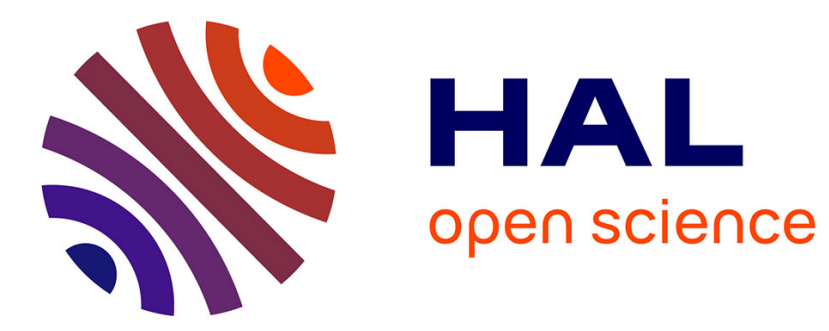

\title{
Radiative heat transfer in strongly forward scattering media using the discrete ordinates method
}

Pedro Granate, Pedro J Coelho, Maxime Roger

\section{To cite this version:}

Pedro Granate, Pedro J Coelho, Maxime Roger. Radiative heat transfer in strongly forward scattering media using the discrete ordinates method. Journal of Quantitative Spectroscopy and Radiative Transfer, 2016, 10.1016/j.jqsrt.2015.12.011 . hal-01282969

\section{HAL Id: hal-01282969 \\ https://hal.science/hal-01282969}

Submitted on 4 Mar 2016

HAL is a multi-disciplinary open access archive for the deposit and dissemination of scientific research documents, whether they are published or not. The documents may come from teaching and research institutions in France or abroad, or from public or private research centers.
L'archive ouverte pluridisciplinaire HAL, est destinée au dépôt et à la diffusion de documents scientifiques de niveau recherche, publiés ou non, émanant des établissements d'enseignement et de recherche français ou étrangers, des laboratoires publics ou privés. 


\title{
RADIATIVE HEAT TRANSFER IN STRONGLY FORWARD SCATTERING MEDIA USING THE DISCRETE ORDINATES METHOD
}

\author{
Pedro Granate*, Pedro J. Coelho ${ }^{* \S}$ and Maxime Roger ${ }^{* *}$ \\ * LAETA, IDMEC, Dept. of Mechanical Engineering, Instituto Superior Técnico, \\ Universidade de Lisboa, Av. Rovisco Pais, 1049-001 Lisboa, Portugal \\ ** Université de Lyon, INSA de Lyon, CETHIL, UMR5008, F-69621 Villeurbanne Cedex \\ ${ }^{\S}$ Correspondence author: \\ Phone: $+351-218418194$ \\ Email: pedro.coelho@tecnico.ulisboa.pt
}




\begin{abstract}
The discrete ordinates method (DOM) is widely used to solve the radiative transfer equation, often yielding satisfactory results. However, in the presence of strongly forward scattering media, this method does not generally conserve the scattering energy and the phase function asymmetry factor. Because of this, the normalization of the phase function has been proposed to guarantee that the scattering energy and the asymmetry factor are conserved. Various authors have used different normalization techniques. Three of these are compared in the present work, along with two other methods, one based on the finite volume method (FVM) and another one based on the spherical harmonics discrete ordinates method (SHDOM). In addition, the approximation of the Henyey-Greenstein phase function by a different one is investigated as an alternative to the phase function normalization. The approximate phase function is given by the sum of a Dirac delta function, which accounts for the forward scattering peak, and a smoother scaled phase function. In this study, these techniques are applied to three scalar radiative transfer test cases, namely a three-dimensional cubic domain with a purely scattering medium, an axisymmetric cylindrical enclosure containing an emitting-absorbing-scattering medium, and a three-dimensional transient problem with collimated irradiation. The present results show that accurate predictions are achieved for strongly forward scattering media when the phase function is normalized in such a way that both the scattered energy and the phase function asymmetry factor are conserved. The normalization of the phase function may be avoided using the FVM or the SHDOM to evaluate the in-scattering term of the radiative transfer equation. Both methods yield results whose accuracy is similar to that obtained using the DOM along with normalization of the phase function. Very satisfactory predictions were also achieved using the delta-M phase function, while the delta-Eddington phase function and the transport approximation may perform poorly.
\end{abstract}


Keywords: Discrete ordinates method; Finite volume method; Spherical harmonics discrete ordinates method; Forward scattering media; Phase function normalization 


\section{INTRODUCTION}

The discrete ordinates method (DOM) was initially developed by Chandrasekhar [1] in his work about interstellar radiation. A few years later, Carlson and Lathrop [2] applied the method to the neutron transport theory. There were some early attempts to use the method to solve thermal radiation problems, but it did not become popular until the last quarter of the 20th century, with the pioneering work of Fiveland [3]. Subsequent developments include improved spatial and angular discretization schemes, generalization to more complex grid structures and to complex enclosures, extensions to non-grey media, media with variable refractive index, and transient problems, as well as parallel implementation [4]. Although the accuracy achieved using the DOM is often satisfactory and the computational requirements moderate, it is well known that errors due to ray effects and false scattering are generally present [5-7]. Another source of error arises in strongly anisotropic media, as a result of lack of conservation of scattered energy [8-9] and/or asymmetry factor of the phase function [10]. This is the subject of the present work, which is concentrated on scalar radiative transfer. Although the focus is on thermal radiation the methods discussed here may be applied to other fields, for example atmospheric radiation, astrophysics, neutron transport, etc.

When the DOM is used, the in-scattering term of the radiative transfer equation (RTE) is approximated by a quadrature over all discrete directions, and there is no guarantee that the integral of the scattering phase function over a spherical surface of unity radius yields $4 \pi$, as it should if there were no approximation. A simple normalization of the scattering phase function to enforce conservation of the scattering energy has been proposed a long time ago by Kim and Lee [9] and implemented by Liu et al. [11]. However, it was found that this method only yields accurate results for small or moderate asymmetry factors of the phase function [10]. When the asymmetry factor increases, the normalization method changes the 
overall shape of the scattering phase function. As a consequence, even though the scattered energy is conserved, the asymmetry factor is not, and this may yield large errors in the case of highly anisotropic phase functions, which become larger in the case of optically thick media. Boulet et al. [12] found that this problem is largely overcome using the finite volume method (FVM) instead of the DOM. The effectiveness of the FVM to surmount this problem has also been found in problems with collimated irradiation [13].

A different normalization procedure, which conserves both the scattered energy and the asymmetry factor for the Henyey-Greenstein phase function, was proposed by Hunter and Guo [10]. This method produced results that closely agree with FVM predictions in an axisymmetric cylindrical enclosure [10], and Monte Carlo benchmark solutions in a cubic enclosure [14]. The application to a Legendre scattering phase function in ultrafast radiative transfer is reported in [15]. A drawback of this normalization procedure is the need to predetermine a normalization matrix. This may be avoided using a simpler normalization technique [16], which simultaneously conserves the scattered energy and the asymmetry factor of the phase function, and maintains most of the phase-function shape. The method was applied to the Henyey-Greenstein phase function, yielding results similar to those obtained using the previous normalization technique, but with a lower computational effort. This technique has recently been extended to collimated radiation for Henyey-Greenstein or Legendre scattering phase-functions [17].

It is also possible to approximate a strongly forward scattering phase function by another approximate phase function where the forward scattering peak is dealt with a Dirac delta function, and the remaining scattering contribution is accounted for using an isotropic or a moderate anisotropic phase function. In such a case, the lack of conservation of scattered 
energy or asymmetry factor is largely overcome.

In the present work, the normalization methods of Liu et al. [11] and Hunter and Guo [10, 16] are applied to three test problems along with two other methods that do not rely on the normalization of the phase function. One of these methods is the FVM, which is applied here only to the in-scattering term. This means that the DOM is still employed, except in the evaluation of that term, in contrast to previous works where the angular discretization of the RTE was fully carried out using the FVM. In this sense, the present approach is a DOM-FVM hybrid formulation. The other method is based on the spherical harmonics discrete ordinates method, which was proposed by Evans in the framework of atmospheric radiation [18]. We are not aware of previous application of this method to thermal radiation problems. The approximation of a strongly scattering forward phase function by a Dirac delta function plus a smooth scattering contribution will be also investigated.

\section{MATHEMATICAL FORMULATION}

The transient RTE for an emitting-absorbing-scattering grey medium may be written as follows [19]:

$$
\frac{1}{c} \frac{\partial I(\mathbf{r}, \mathbf{s}, t)}{\partial t}+\mathbf{s} \cdot \nabla I(\mathbf{r}, \mathbf{s}, t)=-\beta I(\mathbf{r}, \mathbf{s}, t)+\kappa I_{b}(\mathbf{r})+\frac{\sigma_{s}}{4 \pi} \int_{4 \pi} I\left(\mathbf{r}, \mathbf{s}^{\prime}, t\right) \Phi\left(\mathbf{s}^{\prime}, \mathbf{s}\right) d \Omega^{\prime}
$$

where $I(\mathbf{r}, \mathbf{s}, t)$ is the radiation intensity in direction $\mathbf{s}, \mathbf{r}$ is the position vector, $t$ is the time, $c$ is the speed of light in vacuum, $I_{b}$ is the blackbody radiation intensity, $\kappa, \beta$ and $\sigma_{s}$ are the absorption, extinction and scattering coefficients of the medium, respectively, and $\Phi\left(\mathbf{s}^{\prime}, \mathbf{s}\right)$ is the scattering phase function. The boundary condition for a grey surface that emits and reflects diffusely is given by [19]: 
$I\left(\mathbf{r}_{w}, \mathbf{s}, t\right)=\varepsilon I_{b}\left(\mathbf{r}_{w}\right)+\frac{\rho}{\pi} \int_{\mathbf{n} \cdot \mathbf{s}^{\prime}<0} I\left(\mathbf{r}_{w}, \mathbf{s}^{\prime}, t\right)\left|\mathbf{n} \cdot \mathbf{s}^{\prime}\right| d \Omega^{\prime}$

where $I\left(\mathbf{r}_{w}, \mathbf{s}, t\right)$ and $I\left(\mathbf{r}_{w}, \mathbf{s}^{\prime}, t\right)$ are the radiation intensities at boundary point $\mathbf{r}_{w}$ that leave the boundary along $\mathbf{s}$ direction and arrive along $\mathbf{s}^{\prime}$ direction, respectively, $I_{b w}$ is the blackbody radiation intensity at the temperature of the boundary surface, $\varepsilon$ is the surface emissivity, $\rho$ is the surface reflectivity, and $\mathbf{n}$ is the unit vector normal to the surface and pointing into the medium. When collimated radiation is present, the RTE is decomposed into a diffuse and a collimated component, as described in Modest [19].

In the present work, the DOM was used to solve equation (1). The spatial discretization was performed using the CLAM scheme, and the angular discretization was carried out using the $\mathrm{S}_{\mathrm{N}}$ quadrature, except in the cases identified below. In the case of transient problems, the time discretization was achieved using a second-order Runge-Kutta method. Details on the discretization procedure may be found elsewhere [20]. When the DOM is employed, the angular integrals are approximated by quadratures, e.g., the in-scattering term is evaluated as

$\frac{1}{4 \pi} \int_{4 \pi} I\left(\mathbf{r}, \mathbf{s}^{\prime}, t\right) \Phi\left(\mathbf{s}^{\prime}, \mathbf{s}\right) d \Omega^{\prime}=\frac{1}{4 \pi} \sum_{j=1}^{N_{d}} I\left(\mathbf{r}, \mathbf{s}_{j}, t\right) \Phi\left(\mathbf{s}_{i}, \mathbf{s}_{j}\right) w_{j} \quad$ for $i=1,2, \ldots, N_{d}$

where $N_{d}$ is the number of discrete directions and $w_{j}$ is the quadrature weight for the $j$ th direction. The scattering phase function is defined such that the integral of the phase function is equal to $4 \pi$, but the approximation introduced by the quadrature generally yields an error, i.e., the scattered energy is not exactly conserved. The methods used in the present work to address this problem are described below. 


\subsection{Normalization of Kim and Lee [9]}

The technique proposed by Kim and Lee [9], which will be referred hereafter as KL, is straightforward, and consists in multiplying the phase function by a normalization coefficient, so that the new phase function, $\tilde{\Phi}$, is given by:

$$
\tilde{\Phi}\left(\mathbf{s}_{i}, \mathbf{s}_{j}\right)=\Phi\left(\mathbf{s}_{i}, \mathbf{s}_{j}\right) \times\left[\frac{1}{4 \pi} \sum_{j=1}^{N_{d}} \Phi\left(\mathbf{s}_{i}, \mathbf{s}_{j}\right) w_{j}\right]^{-1}
$$

The scattering phase function $\Phi$ in the in-scattering term of equation (1) is replaced by the normalized one, $\tilde{\Phi}$, and this guarantees that the discrete form of the integral on the left side of equation (3) is indeed equal to $4 \pi$ when the radiation intensity is equal to unity for all directions.

\subsection{Normalization of Hunter and Guo[10]}

Boulet et al. [12] noticed that the KL normalization procedure may lead to strong modifications in the values of the discretized phase function when the asymmetry factor is large, and this compromises the accuracy of the results. Hunter and Guo [10] suggested that this may be avoided by enforcing conservation of the asymmetry factor of the phase function, $g$, in addition to conservation of the scattered energy. This is achieved by using a normalized phase function, $\tilde{\Phi}$, such that the following conditions hold:

$$
\frac{1}{4 \pi} \sum_{j=1}^{N_{d}} \tilde{\Phi}\left(\mathbf{s}_{i}, \mathbf{s}_{j}\right) w_{j}=1 \quad \text { for } i=1,2, \ldots, N_{d}
$$


$\frac{1}{4 \pi} \sum_{j=1}^{N_{d}} \tilde{\Phi}\left(\mathbf{s}_{i}, \mathbf{s}_{j}\right)\left(\mathbf{s}_{i} \cdot \mathbf{s}_{j}\right) w_{j}=g \quad$ for $i=1,2, \ldots, N_{d}$

The normalized phase function is defined as follows:

$\tilde{\Phi}\left(\mathbf{s}_{i}, \mathbf{s}_{j}\right)=\tilde{\Phi}\left(\mathbf{s}_{j}, \mathbf{s}_{i}\right)=\left(1+A_{i j}\right) \Phi\left(\mathbf{s}_{i}, \mathbf{s}_{j}\right)$

There are $2 N_{d}$ equations (equations 5 and 6) to be satisfied and $N_{d}\left(N_{d}+1\right) / 2$ unknows (equation 7), so that the system of equations has an infinite number of solutions for the normalization coefficients $A_{i j}$. These coefficients were determined using the least norm solution via QR factorization [21], where $\mathbf{R}$ is an upper triangular nonsingular $\left(2 N_{d} \times 2 N_{d}\right)$ matrix and $\mathbf{Q}$ is an orthogonal $N_{d}\left(N_{d}+1\right) / 2 \times 2 N_{d}$ matrix, i.e., $\mathbf{Q}^{\mathrm{T}} \mathbf{Q}$ is equal to the identity matrix of rank $2 N_{d}$. Hereafter, this normalization procedure will be referred to as HG1.

\subsection{Normalization of Hunter and Guo[16]}

A simpler normalization procedure has been proposed by Hunter and Guo [16], which will be referred to as HG2. In this method, equations (5) and (6) are still enforced, but only the forward and the backward terms of the scattering phase function are normalized, while the others remain unchanged. Hence, equation (7) is only used when $\mathbf{s}_{i \cdot} \mathbf{s}_{j}=1$ or $\mathbf{s}_{i \cdot} \cdot \mathbf{s}_{j}=-1$. This leads to a number of equations equal to the number of unknown $A_{i j}$ coefficients, which may be easily solved analytically after inserting equation (7) into equations (5) and (6), yielding explicit expressions for those coefficients (see [16] for details).

\subsection{Finite volume method}

An alternative to the previous normalization techniques of the scattering phase function is to replace the quadrature used in the DOM for the calculation of the integral on the left side of 
equation (3) by the integration procedure used in the finite volume method, which may be expressed as follows:

$\frac{1}{4 \pi} \int_{4 \pi} I\left(\mathbf{r}, \mathbf{s}^{\prime}, t\right) \Phi\left(\mathbf{s}^{\prime}, \mathbf{s}\right) d \Omega^{\prime}=\frac{1}{4 \pi} \sum_{j=1}^{N_{d}} I_{j} \bar{\Phi}_{i j} \Delta \Omega_{j} \quad$ for $i=1,2, \ldots, N_{d}$

with $\bar{\Phi}_{i j}$ defined as follows:

$\bar{\Phi}_{i j}=\frac{\int_{\Delta \Omega_{i}} \int_{\Delta \Omega_{j}} \Phi\left(\mathbf{s}^{\prime}, \mathbf{s}\right) d \Omega^{\prime} d \Omega}{\Delta \Omega_{i} \Delta \Omega_{j}}$

The integrals on the right hand side may be evaluated analytically if the scattering phase function is simple enough. In the general case, however, a numerical integration is required, causing a numerical integration error, and preventing the scattering energy from being exactly conserved. This error may be greatly reduced by splitting the control angles $\Delta \Omega_{i}$ and $\Delta \Omega_{j}$ into smaller angles. In the present work, an adaptive integration method [22] was used for the quadruple integration in equation $8(\mathrm{~b})$, with a prescribed relative error of $10^{-4}$. This guarantees that the integration error is also negligible for the vast majority of problems. In order to apply this method, the boundaries of the control angles must be defined, and therefore the $\mathrm{S}_{\mathrm{N}}$ quadrature cannot be used. Instead, the polar /azimuthal discretization commonly used in the FVM has been employed.

\subsection{Spherical harmonics discrete ordinates method}

The last method used in the present work to evaluate the in-scattering term of the RTE is based on the spherical harmonics discrete ordinates method introduced by Evans [18] in the field of atmospheric radiation. It will be referred to below as SHDOM. This method is rather different from the standard DOM, but the only feature of the SHDOM that we will retain here 
is in the evaluation of the in-scattering term, while the standard DOM is used elsewhere. This term is evaluated using spherical harmonics. Denoting the in-scattering term of the RTE by $S_{\mathrm{SH}}(\mathbf{r}, \mathbf{s})$, where the temporal dependence was omitted for simplicity, the following truncated expansion in spherical harmonics is taken:

$S_{\mathrm{SH}}(\mathbf{r}, \mathbf{s})=\frac{\sigma_{s}}{4 \pi} \int_{4 \pi} I\left(\mathbf{r}, \mathbf{s}^{\prime}\right) \Phi\left(\mathbf{s}^{\prime}, \mathbf{s}\right) d \Omega^{\prime}=\sum_{l=0}^{L} \sum_{m=-M}^{M} Y_{l}^{m}(\mathbf{s}) S_{l}^{m}(\mathbf{r})$

In this equation, $Y_{l}^{m}(\mathbf{s})$ are spherical harmonics, $S_{l}^{m}(\mathbf{r})$ are coefficients that depend only on the spatial coordinates, and $M \leq L$. Note that terms such that $M>l$ are equal to zero. The real spherical harmonics are defined as follows:

$Y_{l}^{m}(\mathbf{s})=\Lambda_{l}^{m}(\mu) u(m \phi)=\sqrt{\frac{2 l+1}{2 \pi\left(1+\delta_{m o}\right)} \frac{(l-|m|) !}{(l-|m|) !}} P_{l}^{|m|}(\mu) u(m \phi)$

where $\Lambda_{l}^{m}$ are associated Legendre functions, $P_{l}^{|m|}(\mu)$ are associated Legendre polynomials, and $u(m \phi)=\cos (m \phi)$ if $m \geq 0$ and $u(m \phi)=\sin (m \phi)$ if $m<0$. The in-scattering term is transformed to discrete ordinates as follows:

$S_{\mathrm{DOM}}\left(\mathbf{r}, \mathbf{s}_{j k}\right)=\sum_{l=0}^{L} \sum_{m=-M}^{M} \Lambda_{l}^{m}\left(\mu_{j}\right) u\left(m \phi_{k}\right) S_{l}^{m}(\mathbf{r})$

where $\mu_{j}=\cos \theta_{j}$ and subscripts $j$ and $k$ identify one among the $N_{d}$ directions of the DOM angular discretization. The RTE is solved using the in-scattering term given by equation (11) to obtain the radiation intensity for every direction, namely $I_{j k}(\mathbf{r})=I\left(\mathbf{r}, \mathbf{s}_{j k}\right)$.

The radiation intensity at every grid node, evaluated from the DOM, is transformed to 
spherical harmonics space as follows:

$I_{\mathrm{SH}}(\mathbf{r}, \mathbf{s})=\sum_{l=0}^{L} \sum_{m=-M}^{M} Y_{l}^{m}(\mathbf{s}) I_{l}^{m}(\mathbf{r})$

The coefficients $I_{l}^{m}(\mathbf{r})$ are given by [18]:

$$
\begin{aligned}
I_{l}^{m}(\mathbf{r}) & =\int_{4 \pi} Y_{l}^{m}(\mathbf{s}) I(\mathbf{r}, \mathbf{s}) d \Omega=\int_{4 \pi} \Lambda_{l}^{m}(\mu) u(m \phi) I(\mathbf{r}, \mathbf{s}) d \Omega \\
& =\sum_{j=1}^{N_{\mu}} \sum_{k=1}^{N_{\phi}} \omega_{j} \omega_{k} \Lambda_{l}^{m}\left(\mu_{j}\right) u\left(m \phi_{k}\right) I\left(\mathbf{r}, \mathbf{s}_{j k}\right)
\end{aligned}
$$

where $N_{\mu}$ and $N_{\phi}$ are the number of cosine polar angles and the number of azimuthal angles, respectively, and $\omega_{j}$ and $\omega_{k}$ are the quadrature weights. The values of $N_{\mu}$ and $N_{\phi}$ are related to $L$ and $M$ according to the following relations [18], which ensure that the spherical harmonics are orthogonal when their product is integrated:

$$
\begin{aligned}
& L=N_{\mu}-1 \\
& M=N_{\phi} / 2-1
\end{aligned}
$$

In addition, since $M$ must be lower or equal than $L$, the following relation also holds: $N_{\phi} \leq 2 N_{\mu}$. A Gauss-Legendre quadrature was used for the integration in the polar angle, i.e., the cosines of the polar angles of the discrete directions in the DOM, $\mu_{j}$, correspond to the roots of the Legendre polynomial of order equal to that of the quadrature, i.e., $N_{\mu}$. The azimuthal angles are evenly spaced, and the quadrature weights $\omega_{k}$ are equal to $2 \pi / N_{\phi}$.

The scattering phase function written in terms of Legendre polynomials may also be 
expressed in terms of truncated spherical harmonics:

$\Phi(\cos \Theta)=\sum_{l=0}^{L} \chi_{l} P_{l}(\cos \Theta)=\sum_{l=0}^{L} \chi_{l} \frac{4 \pi}{2 l+1} \sum_{m=-l}^{l} Y_{l}^{m}(\mathbf{s}) Y_{l}^{m}\left(\mathbf{s}^{\prime}\right)$

where $\cos \Theta=\mathbf{s} \cdot \mathbf{s}^{\prime}$ and $P_{l}$ is the Legendre polynomial of order $l$. The Henyey-Greenstein phase function may be written in terms of Legendre polynomials by setting $\chi_{l}=(2 l+1) g^{l}$. The in-scattering term is evaluated in spherical harmonics space as follows:

$$
\begin{aligned}
S_{\mathrm{SH}}(\mathbf{r}, \mathbf{s}) & =\frac{\sigma_{s}}{4 \pi} \int_{4 \pi} I\left(\mathbf{r}, \mathbf{s}^{\prime}\right) \Phi\left(\mathbf{s}^{\prime}, \mathbf{s}\right) d \Omega^{\prime} \\
& =\frac{\sigma_{s}}{4 \pi} \int_{4 \pi}\left(\sum_{l=0}^{L} \sum_{m=-M}^{M} Y_{l}^{m}\left(\mathbf{s}^{\prime}\right) I_{l}^{m}(\mathbf{r})\right)\left(\sum_{i=0}^{L} \chi_{i} \frac{4 \pi}{2 i+1} \sum_{j=-i}^{i} Y_{i}^{j}(\mathbf{s}) Y_{i}^{j}\left(\mathbf{s}^{\prime}\right)\right) d \Omega^{\prime} \\
& =\sigma_{s} \sum_{l=0}^{L} \sum_{m=-M}^{M} \frac{\chi_{l}}{2 l+1} Y_{l}{ }^{m}(\mathbf{s}) I_{l}^{m}(\mathbf{r})
\end{aligned}
$$

where the orthogonality property of the spherical harmonics has been used. It follows from equations (9) and (17) that the coefficients of the spherical harmonics expansion of the inscattering term are given by:

$S_{l}^{m}(\mathbf{r})=\sigma_{s} \frac{\chi_{l}}{2 l+1} I_{l}^{m}(\mathbf{r})$

The solution algorithm may be summarized as follows:

(i) The in-scattering source term evaluated in the spherical harmonics space is transformed to discrete ordinates according to equation (11). This step is ignored in the first iteration.

(ii) The RTE is solved using the DOM, with the in-scattering term evaluated in step (i). 
In the first iteration, the in-scattering term is calculated using a quadrature, as in the standard DOM.

(iii) The radiation intensity field calculated in step (ii) is transformed to spherical harmonics according to equation (12), with the coefficients given by equation (13). Only these coefficients need to be calculated.

(iv) The in-scattering term in spherical harmonics space is calculated from the radiation intensity field in spherical harmonics using equation (17). Only the coefficients $S_{l}^{m}(\mathbf{r})$, given by equation (18), of the spherical harmonics expansion of the in-scattering term, which is expressed by equation (9), need to be evaluated. This involves simple multiplications rather than summations that typically appear in quadrature rules.

\subsection{Approximate scattering phase function}

In addition to the methods described above, which are applied to the Henyey-Greenstein scattering phase function of the problem under consideration, we will further examine the approximation of the Henyey-Greenstein phase function using the transport, the deltaEddington or the delta-M approximations. In all cases, the approximate scattering phase function is defined as follows:

$\Phi(\cos \Theta)=2 f \delta(1-\cos \Theta)+(1-f) \Phi^{*}(\cos \Theta)$

In the transport approximation [23], the phase function $\Phi^{*}$ is equal to unity and the parameter $f$ is set equal to the asymmetry factor. If the transient term of RTE is neglected, then the transport approximation is equivalent to replace the anisotropic scattering problem by an isotropic one with the following modified scaled parameters: 
$\hat{\omega}=\frac{\omega(1-f)}{1-\omega f}$

$\hat{\tau}=(1-\omega f) \tau$

In these equations, $\omega$ and $\tau$ stand for the albedo and optical thickness, respectively, and the circumflex denotes a scaled parameter. In the delta-Eddington approximation, the phase function $\Phi^{*}$ is defined as follows [24]:

$\Phi^{*}(\cos \Theta)=1+3 g^{\prime} \cos \Theta$

where parameters $f$ and $g^{\prime}$ are given by:

$$
\begin{aligned}
& f=g^{2} \\
& g^{\prime}=\frac{g-f}{1-f}
\end{aligned}
$$

In both cases, the DOM may be used without normalization of the approximated phase function, since the strong forward scattering peak is fully accounted for by the Dirac delta function, and the remaining term of the phase function is smooth, no matter the value of the asymmetry factor.

The delta-M phase function is defined as follows [25]:

$$
\Phi^{*}(\cos \Theta)=\sum_{n=0}^{M}(2 n+1) \frac{g^{n}-f}{1-f} P_{n}(\cos \Theta)
$$

where parameter $f$ is given by $f=g^{2 M}$. In the case of $M=1$, the delta-Eddington approximation is recovered. When $M$ increases, the delta-M phase function becomes closer to the HenyeyGreenstein phase function and the contributions of the first and second terms on the right side 
of equation (19) are smaller and larger, respectively. Therefore, the asymmetry factor of $\Phi^{*}$ increases. If $M$ is small enough, the normalization of the phase function is not needed. Otherwise, it becomes necessary. However, in such a case there is no advantage in the replacement of the Henyey-Greenstein by the delta-M phase function.

\section{RESULTS AND DISCUSSION}

Three test cases are presented in this section. The first two are steady-state radiative transfer problems, one in a 3D cubical domain and the other in a 2D axisymmetric enclosure. The last test case addresses a transient problem with collimated incident radiation. The methods outlined above are compared. Their acronyms are summarized here to allow easier identification:

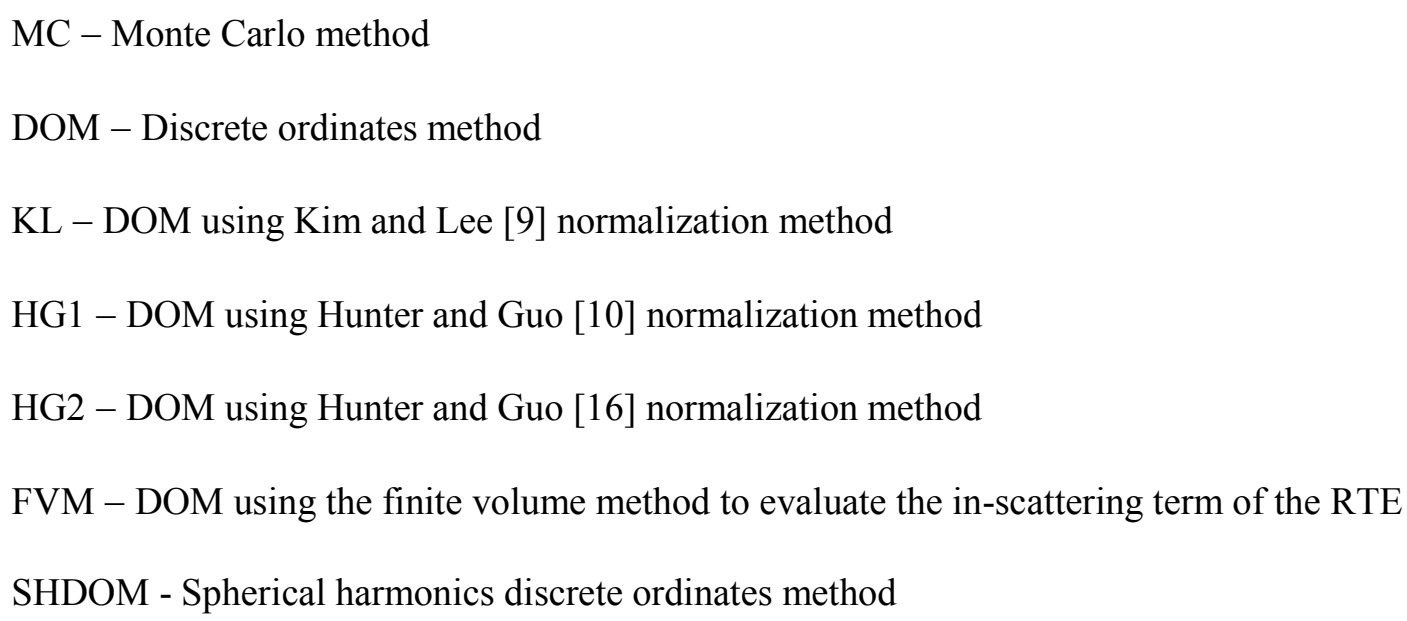

\subsection{Test case 1}

The first test case consists of a cube of unit side length, $L$, containing a grey and cold (nonemitting) medium with an optical thickness $\tau=\beta L=10$. The walls are black and cold, except the bottom one $(z=0)$, which has a unit emissive power. The scattering albedo is $\omega=\sigma_{s} / \beta=1$, and the Henyey-Greenstein phase function was considered. The calculations were carried out using a uniform mesh with $25 \times 25 \times 25$ grid nodes and an $\mathrm{S}_{12}$ quadrature for the DOM and the KL, HG1 and HG2 normalization methods. A quadrature with 7 polar angles and 3 azimuthal angles per octant was used in the case of the FVM, yielding the same number of discrete 
directions as the $\mathrm{S}_{12}$. In the case of the SHDOM, $N_{\mu}$ and $N_{\phi}$ were set to 14 and 12 , respectively, yielding again the same number of discrete directions.

This test case was also used by Boulet et al. [12] and Hunter and Guo [14, 16]. Boulet et al. [12] used the FVM for the spatial and angular discretization of the RTE, along with the STEP scheme, a slightly coarser mesh $(21 \times 21 \times 21)$ and a little finer angular discretization with 4 polar angles and 6 azimuthal angles per octant. Hunter and Guo [14] employed the DOM with the same spatial and angular discretizations used in the present work, but employed the positive scheme instead of the CLAM scheme. Our results are very similar to those obtained in [12] and [14]. The results presented below are compared with a Monte Carlo (MC) solution also reported in [12].

Figure 1 shows the influence of the grid size and quadrature on the incident heat flux, normalized by the emissive power of the hot boundary, along a central line on the top boundary of the cubical enclosure. The plotted results were calculated using the DOM with the HG2 normalization method of the phase function, for an asymmetry factor equal to 0.93 . It can be seen that the grid size has a small influence on the results. The predictions obtained using the $\mathrm{S}_{4}$ quadrature are rather different from all the others, but the $\mathrm{S}_{12}$ and $\mathrm{S}_{16}$ give results similar to each other, while the results of the $S_{8}$ quadrature depart a little from them. Therefore, the mesh with $25 \times 25 \times 25$ grid nodes and the $S_{12}$ quadrature are adequate for the present test case.

A comparison of the various methods used to handle the strong anisotropy of the phase function is displayed in figure 2 . When the asymmetry factor is equal to 0.20 , all methods yield similar results, which closely match the Monte Carlo reference solution. In fact, 
normalization of the scattering phase function is not needed in this case. If the asymmetry factor is equal to 0.80 , the KL method overestimates by up to $7 \%$ the predictions of all other methods, which are in good agreement with each other and with the Monte Carlo reference solution. In the case of an asymmetry factor of 0.93 , the KL results are rather poor. This is consistent with previous works $[12,14]$, and is explained by the change of shape of the scattering phase function, due to the normalization introduced to enforce conservation of the scattered energy. The asymmetry factor is modified due to this normalization, leading to the poor predictions observed. This problem is enhanced with the increase of the asymmetry factor. The remaining four methods employed here successfully overcome the limitations of the KL method, even though their predictions are not as close to the MC solution as for the lower values of the asymmetry factor. The HG1 and HG2 normalization methods yield very similar results, which underpredict by 8 to $10 \%$ the MC benchmark solution, in agreement with the results reported in the literature $[14,16]$. Both the SHDOM and the FVM perform very satisfactorily, and their predictions are even closer to the $\mathrm{MC}$ solution than those of the HG1 and HG2 methods. The very good performance of the FVM is consistent with the findings of Boulet et al. [12].

In general, the computational requirements of the SHDOM are the lowest, even though the need to switch between the discrete ordinates and the spherical harmonics spaces might suggest the opposite. Note, however, that the calculation of the in-scattering term in the spherical harmonics space only requires the evaluation of the coefficients of the spherical harmonics expansion by means of equation (18), which involves a straightforward multiplication. In the case of $g=0.20$, the CPU time for SHDOM was about $600 \mathrm{~s}$ in a personal computer with an Intel ${ }^{\circledR}$ Core $^{\mathrm{TM}}$ i7-4700HQ @ 2.4 GHz processor with 8Gb RAM, compared with about $950 \mathrm{~s}$ for the other methods. When $g=0.80$, the CPU time for SHDOM was about 
$300 \mathrm{~s}$, compared with $370-420 \mathrm{~s}$ for the other methods. Finally, in the case of $g=0.93$, the CPU time for the SHDOM was about $360 \mathrm{~s}$, exceeding a little the computational requirements of the FVM ( 300s), but remaining lower than those of KL ( $\sim 540 \mathrm{~s})$, HG1 ( $\sim 840 \mathrm{~s})$ and HG2 ( $\sim 1030$ s). The CPU time for the normalization procedure has not included, but it is marginal (less than 10s for $\mathrm{HG} 1$, and negligible for KL and $\mathrm{HG} 2$ ).

The decrease of CPU time when $g$ increases from 0.20 to 0.80 is likely to be due to the fact that, in the former case, coupling between the different directions is stronger. When the asymmetry factor increases, only the directions closer to the direction of propagation play a role, while the remaining directions have a negligible contribution to the in-scattering term. However, when the asymmetry factor approaches unity, the contribution to the in-scattering term of directions close to the direction of propagation becomes increasingly larger, and since directions different from that of propagation are treated implicitly, the number of iterations required to achieve convergence is likely to increase. This may explain the increase of CPU time observed for most methods when $g$ increases from 0.80 to 0.93 .

\subsection{Test case 2}

In the second test case, an axisymmetric cylindrical enclosure with radius $R=1 \mathrm{~m}$ and height $H=2 \mathrm{~m}$ is considered. The walls are black and cold, except the cylindrical surface, which has an emissive power of unity. The medium is cold, and scatters according to the HenyeyGreenstein phase-function. The calculations were performed using a uniform mesh with $25 \times 25$ control volumes. The $\mathrm{S}_{16}$ quadrature was used for the DOM and the KL, HG1 and HG2 normalization methods, while 9 polar and 4 azimuthal angles per octant were used in the FVM. In the case of the SHDOM, $N_{\mu}$ and $N_{\phi}$ were set to 18 and 16, respectively. Hence, the 
same number of discrete directions is used for all methods. We first consider a purely scattering medium with an optical thickness, based on the radius of the cylinder, equal to 25 .

This test case was proposed by Hunter and Guo [10], who compared the FVM and DOM using the same grid size and angular discretization considered here. The results obtained using the STEP scheme, which are not shown here, closely match those reported in [10] when the phase function is normalized according to HG1 method. When the CLAM scheme is employed, the predicted net heat fluxes on the hot boundary are lower than those obtained using the STEP scheme, particularly for the lowest value of the asymmetry factor (0.8).

Figure 3 shows a comparison between the net heat fluxes on the hot wall, normalized by its emissive power, obtained for the mesh and quadrature mentioned above, and for a much finer mesh with $100 \times 100$ control volumes and angular discretization, with 12 polar angle and 12 azimuthal angle subdivisions per octant. The DOM was used along with the CLAM scheme and the HG1 method. The figure reveals a very low sensitivity of the results obtained to the spatial and angular grid refinement. Hence, the results displayed in figure 4, where the different methods are compared, were obtained using the coarsest discretization.

Figure 4 shows that all methods perform similarly for $g=0.80$, as far as the accuracy is concerned. When $g$ increases to 0.90 , the solutions obtained using HG1, HG2, FVM and SHDOM are in good agreement with each other. The KL normalization method overestimates the net heat fluxes by about $20 \%$ at $z=H$ in comparison with HG1. In the case $g=0.95$, the differences between the HG1, HG2, FVM and SHDOM remain small. The KL predictions are about $20 \%$ higher than those of the HG1 method in the middle of the cylinder, in agreement with previously reported results [10]. 
Additional calculations were performed for an asymmetry factor of 0.95 , an albedo of 0.90 , and three different values of the optical thickness of the medium. Our predictions obtained using HG1 normalization method are in close agreement with those reported in [10]. The influence of the grid size and angular discretization is relatively small, as evidenced in figure 5, obtained using the same normalization procedure, but the presence of ray effects is visible when the optical thickness of the medium is equal to unity, despite the fine discretizations employed. Figure 6 shows the comparison of the various methods employed for the coarsest discretization. The results obtained using KL, HG1 and HG2 are very close to each other for $\tau=1.0$ and $\tau=5.0$. The FVM and SHDOM yield smooth results for $\tau=1.0$, i.e., ray effects are not visible, and the predictions are in good agreement with those obtained using the DOM for both $\tau=1.0$ and $\tau=5.0$. When the optical thickness increases to $\tau=25.0$, the $\mathrm{KL}$ method overpredicts the DOM solutions for HG1 and HG2 normalization methods by about 5\%, while the FVM underpredicts those DOM solutions by $1.5 \%$. The SHDOM closely matches the DOM predictions.

The CPU times required for the solution of the RTE when the DOM along with HG1 and HG2 normalization methods are used are generally close to each other. The FVM and SHDOM require more CPU time than the DOM (along with HG1 or HG2 methods) for $\tau=25.0$, no matter the asymmetry factor, but lower CPU time for $\tau=1.0$ and $\tau=5.0$, while an opposite trend was found for the DOM along with KL method. Regardless of the method used, the CPU time required to solve the RTE is typically lower than $25 \mathrm{~s}$ for $g=0.95$, and lower than $55 \mathrm{~s}$ for other asymmetry factors, but depends on the optical properties of the medium. These times are much smaller than in test case 1 , because the present problem is two-dimensional. 
The CPU times required for the KL and HG2 normalization of the scattering phase function are negligible, but those of the HG1 normalization procedure (about $110 \mathrm{~s}$ ) and of the evaluation of the average scattering phase function in the FVM (about 170 s), largely exceed the CPU time needed to solve the RTE for the present test case.

\subsection{Test case 3}

In the last test case, a three-dimensional cubic domain of side $L=1 \mathrm{~m}$, containing a scattering and absorbing homogeneous medium, is studied. The medium is subjected to an incident short pulse laser source normal to the boundary $x=0$, while the remaining boundaries are nonreflecting and cold (non-emitting). The medium is also cold, with an optical thickness of 10 and a scattering albedo of 0.5 . The scattering is described again by the Henyey-Greenstein phase function with an asymmetry factor of 0.90 . The collimated intensity of the short pulse laser, denoted by $I_{c}$, is Gaussian in time and uniform in space, according to:

$I_{c}(x=0, y, z, \mathbf{s}, t)=I_{o} \delta(\mathbf{s}-\mathbf{i}) \exp \left[-4(\ln 2)\left(\left(t-3 t_{p}\right) / t_{p}\right)^{2}\right], \quad 0<t<6 t_{p}$

where $\delta$ is the Dirac delta function and $I_{o}$ is the maximum radiative intensity of the pulse, which occurs at $t=3 t_{p}$. The dimensionless time parameter, defined as $t_{p}^{*}=c \beta t_{p}$, with $c$ standing for the speed of light, is set equal to 0.5 . After $t=6 t_{p}$ the medium is free from irradiation. In this test case we are concerned with the transmitted, $T$, and reflected, $R$, temporal signature of the incident radiation, which are given by:

$$
T(t)=\frac{1}{I_{o} L^{2}} \int_{0}^{L} \int_{0}^{L}\left(I_{c}(x=L, y, z, \mathbf{s}, t)+\int_{2 \pi} I_{d}(x=L, y, z, \mathbf{s}, t)(\mathbf{s} \cdot \mathbf{i}) d \mathbf{s}\right) d y d z
$$


$R(t)=\frac{1}{I_{o} L^{2}} \int_{0}^{L} \int_{0}^{L} \int_{2 \pi} I_{d}(x=0, y, z, \mathbf{s}, t)(-\mathbf{s} \cdot \mathbf{i}) d \mathbf{s} d y d z$

where $I_{d}$ is the diffuse component of the radiation intensity.

The temporal discretization was performed using a second-order Runge-Kutta scheme. The domain was discretized using a grid with $200 \times 20 \times 20$ control volumes and an $\mathrm{S}_{12}$ quadrature, and the time step $\Delta t$ was defined as $\Delta t=\alpha . \Delta x / c$, where the stability parameter $\alpha$ was set equal to 0.5 . A previously validated Monte Carlo algorithm [26] was used to provide benchmark results.

The KL and HG1 normalization methods may be readily extended to account for the collimated component [27], as well as the FVM and SHDOM. However, the HG2 normalization procedure is not directly applicable, since the collimated component does not belong to the set of discrete directions, and therefore it is not feasible to normalize the forward and backward components of the phase function. However, the original idea underlying this method may be maintained by applying the normalization to the discrete directions with the scattering angle closest to $0^{\circ}$ and $180^{\circ}$, as described in [17]. A drawback of this implementation is the possible occurrence of unphysical negative values of the normalized scattering phase function for a scattering angle close to $180^{\circ}$, as reported in [17] and confirmed in the present test case. Despite this, it was possible to obtain a converged solution, due to the minor role played by the backward-scattering terms in the case of a large and positive asymmetry factor.

The results obtained are displayed in figure 7. If the DOM is used without any normalization method for the phase function, the transmittance is overestimated by more than one order of 
magnitude, while the decay of the reflectance after the peak is also strongly overestimated. Even though all other methods yield much better solutions, the discrepancies among them, and in comparison with the Monte Carlo reference solution, are larger than for the previous test cases. The KL normalization procedure provides a surprisingly good estimation of the transmittance, but does not perform well as far as the reflectance calculation is concerned. The HG1 and HG2 methods underpredict the peak of the transmittance by about $15 \%$, but match well the reference solution for shorter and longer times. The HG1 method further yields an accurate prediction of the reflectance, while the HG2 procedure does not, due to the problem with the normalization of the collimated radiation component mentioned above. The FVM predictions of the transmittance are similar to those of the DOM with HG1 or HG2 methods. The former method marginally overpredicts the peak of reflectance, in contrast with the latter, which slightly underestimates that peak, in comparison with the MC reference solution. The SHDOM provides the best estimate of the transmittance, and predicts well the reflectance.

Both the DOM with any of the tested normalization methods and the FVM require about $26 \mathrm{~h}$ of CPU time for this transient problem, while the SHDOM is far more efficient, and needs only about $11.5 \mathrm{~h}$.

\subsection{Approximation of the scattering phase function}

We will now examine whether the approximation of the Henyey-Greenstein phase function by the phase functions mentioned in section 2.6 is satisfactory or not for the previous test cases. Figures $8(\mathrm{a})$ and $8(\mathrm{~b})$ show the predictions obtained using the DOM for test cases 1 and 2, respectively, and corresponding to the set of radiative properties considered in Figs. 2 and 4, respectively. In the case of the Henyey-Greenstein phase function, the HG1 normalization 
method was used, while no normalization is required for the transport, delta-Eddington and delta-M approximations.

The transport and delta-Eddington approximations perform quite well for asymmetry factors up to 0.9 in the two test cases. Therefore, they can be used instead of the normalization procedures discussed above. The delta-Eddington approximation performs better than the transport approximation, as expected, since $\Phi^{*}$ is linearly anisotropic in the former approximation and isotropic in the latter. The delta-Eddington and delta-M phase functions yield similar results, which are graphically indistinguishable, and therefore only the results calculated using the former phase function are presented.

When the asymmetry factor is greater than 0.90 , the differences between the results for the different phase functions become larger. Although the transport and delta-Eddington approximations remain an attractive option for test case 1, improved results are obtained using the delta-M with $M=2$, denoted as delta-M2 in the figure. The results for delta-M approximation are not shown for test case 2 , since they are almost coincident with those of the delta-Eddington approximation.

In test case 3, however, the results obtained using the transport and delta-Eddington approximations are rather different from those calculated using the Henyey-Greenstein phase function, as illustrated in Figs. 8(c) and 8(d). The transmittance peaks are overestimated by a factor of two or more. Moreover, the maximum transmittance occurs earlier, and the subsequent decay is faster when those approximate phase functions are used. The reflectance peak calculated using the transport approximation is marginally overestimated, and that computed using the delta-Eddington phase function is significantly underestimated. Accordingly, these approximate phase functions should not be used in the case of transient radiative transfer with collimated irradiation. However, the delta-M 
function provides much better results, particularly for $M=6$, even though the transmittance peak occurs slightly earlier in comparison with the solution computed using the Henyey-Greenstein phase function.

Overall, and since the Henyey-Greenstein phase function is also an approximation of an actual phase function, the delta-M may be used instead of the Henyey-Greenstein phase function, allowing the DOM to be applied to strongly forward scattering media without any normalization technique. However, the value of $\mathrm{M}$ in the delta-M approximation needs to be carefully chosen. If it is too small, the approximation may be poor, while if it is too large, the weight of the Dirac delta function in Eq. (19) is small, and the results may be worst. In fact, even though the delta-M function approximates better the Henyey-Greenstein phase function when $M$ increases, the asymmetry factor of $\Phi^{*}$ increases as well. When this asymmetry factor is large enough and the DOM is used, the error in the calculation of the scattered energy and asymmetry factor tends to increase. In such a case, the phase function needs to be normalized to avoid poor predictions, but then there would be no advantage in replacing the Henyey-Greenstein by the delta-M phase function, as pointed out in section 2.6. In test case 3, the results obtained using $M=8$ (not show in the figure) are worst then those computed using $M=6$, because the asymmetry factor of $\Phi^{*}$ for $M=8$ is large $(\mathrm{g}=0.87)$.

\section{CONCLUSIONS}

An investigation of different strategies to deal with highly anisotropic scattering media in the framework of the DOM was carried out. Different proposals for the normalization of the phase function previously reported in the literature were compared with two other approaches that do not rely on the phase function normalization. One of them is based on the finite volume method for the evaluation of the in-scattering term of the RTE, and the other is based on the spherical harmonics discrete ordinates method, formerly used in the field of atmospheric radiation. The use of approximate phase functions given by the sum of a Dirac 
delta function and a smoother phase function is also investigated as an alternative to the normalization of the strongly forward scattering phase function.

The KL normalization procedure does not perform well for highly anisotropic scattering, in agreement with past work, since it does not preserve the asymmetry factor of the phase function. The HG1 method was highly effective for all studied cases, but the computational requirements of the normalization procedure may exceed those needed for the solution of the RTE. The HG2 technique overcomes that drawback, and provides solutions of similar accuracy for diffuse radiation. In the case of collimated radiation, however, this technique may yield negative normalization coefficients and physically unrealistic values of the normalized scattering phase function. These may lead to negative values of the radiation intensity. Even though this did not prevent convergence for the test case selected in this work, the predictions of the reflectance for the transient test case were not satisfactory. Therefore, this normalization procedure should be used with caution for collimated radiation.

The FVM is an interesting alternative to the normalization procedures, since it does not modify the phase function. However, an accurate integration is needed to obtain the average phase function for every pair of discrete directions, before and after scattering, and this may be time-consuming in the case of acute forward scattering. In the present work, an adaptive integration method was employed, which yields satisfactory results for an asymmetry factor up to 0.95 .

The SHDOM performed quite well for all test cases, and the computational cost was the lowest, except in the two-dimensional axi-symmetric problem and for optically thick media. It proved to be a very interesting alternative to the DOM anisotropically scattering media, and deserves further investigation. 
The approximation of the Henyey-Greenstein phase function using the transport or the deltaEddington approximation is very satisfactory when the transient term of the RTE is negligible and the asymmetry factor does not exceed 0.90 , and still performs reasonably for higher asymmetry factors, avoiding the normalization of the phase function. In the case of transient problems with collimated irradiation, those approximations do not perform well, but the more accurate delta-M phase function yielded very satisfactory results. Therefore, it constitutes an interesting alternative that relaxes the need to normalize the phase function when the DOM is applied to strongly forward scattering media. However, the value of $M$ needs to be carefully chosen, and should be as large as possible, provided that the scattered energy and the asymmetry factor are accurately evaluated.

\section{ACKNOWLEDGEMENTS}

This work was financially supported by national funds by FCT-Fundação para a Ciência e Tecnologia under the project PTDC/EMS-ENE/1028/2012, and through IDMEC, under LAETA, project UID/EMS/50022/2013. 


\section{REFERENCES}

[1] Chandrasekhar S. Radiative transfer. London: Oxford University Press; 1950.

[2] Carlson BG, Lathrop KD. Transport theory - The method of discrete ordinates. In: Greenspan H, Kelber CN, Okrent D, editors. Computing methods in reactor physics. New York: Gordon and Breach; 1968, p. 165-266.

[3] Fiveland WA. Discrete-ordinates solutions of the radiative transport equation for rectangular enclosures. J Heat Transfer 1984;106:699-706.

[4] Coelho PJ. Advances in the discrete ordinates and finite volume methods for the solution of radiative heat transfer problems in participating media. J Quant Spectrosc Radiat Transfer 2014;145:121-46.

[5] Chai JC, Lee HS and Patankar SV. Ray Effect and False Scattering in the Discrete Ordinates Method. Numer Heat Transfer B 1993;24:373-89.

[6] Raithby GD. Evaluation of discretization errors in finite-volume radiant heat transfer predictions. Numerical Heat Transfer, Part B 1999;36:241-64.

[7] Coelho PJ. The role of ray effects and false scattering on the accuracy of the standard and modified discrete ordinates methods. J Quant Spectrosc Radiat Transfer 2002;73:231-8.

[8] Wiscombe WJ. On initialization, error and flux conservation in the doubling method. J Quant Spectrosc Radiat Transfer 1976;18:637-58.

[9] Kim TK, Lee H. Effect of anisotropic scattering on radiative heat transfer in twodimensional rectangular enclosures. Int J Heat and Mass Transfer 1988;31:1711-21.

[10] Hunter B, Guo Z. Conservation of asymmetry factor in phase function discretization for 
radiative transfer analysis in anisotropic scattering media. Int $\mathrm{J}$ Heat Mass Transfer 2012;55:1544-52.

[11] Liu LH, Ruan LM, Tan HP. On the discrete ordinates method for radiative heat transfer in anisotropically scattering media. Int J Heat and Mass Transfer 2002:45:3259-62.

[12] Boulet P, Collin A, Consalvi JL. On the finite volume method and the discrete ordinates method regarding radiative heat transfer in acute forward anisotropic scattering media. $\mathrm{J}$ Quant Spectrosc Radiat Transfer 2007;104:460-73.

[13] Collin A, Consalvi JL, Boulet P. Acute anisotropic scattering in a medium under collimated irradiation. Int J Thermal Sciences 2011;50;19-24.

[14] Hunter B, Guo Z. Phase-function normalization in the 3-D discrete-ordinates solution of radiative transfer — Part II: Benchmark comparisons. Numerical Heat Transfer, Part B $2012 ; 62: 223-42$.

[15] Hunter B, Guo Z. Phase-function normalization for accurate analysis of ultrafast collimated radiative transfer. Appl Opt 2012;51:2192-20.

[16] Hunter B, Guo Z. A new and simple technique to normalize the HG phase function for conserving scattered energy and asymmetry factor. Numerical Heat Transfer, Part B $2014 ; 65: 195-217$.

[17] Hunter B, Guo Z. Applicability of phase-function normalization techniques for radiation transfer computation. Numer Heat Transfer B 2015;67:1-24.

[18] Evans KF. The spherical harmonic discrete ordinate method for three-dimensional atmospheric radiative transfer. J Atmos Sci 1998;55:429-46.

[19] Modest MF. Radiative heat transfer. 3rd ed. New York: Academic Press; 2013.

[20] Coelho PJ. Discrete ordinates and finite volume methods: mathematical formulation. 
[21] Golub GH, Van Loan CF. Matrix computations. Baltimore: Johns Hopkins Univ Press; 1996.

[22] Patterson TNL. The optimum addition of points to quadrature formulae. Math Comput $1968 ; 22: 847-56$.

[23] Davison B. Neutron transport theory. London: Oxford University Press; 1957.

[24] Joseph JH, Wiscombe WJ, Weinman JA. The delta-Eddington approximation for radiative flux transfer. J Atmos Sci 1976;33:2452-9.

[25] Wiscombe WJ. The delta-M method. Rapid yet accurate radiative flux calculations for strongly asymmetric phase functions. J Atmos Sci 1977;34:1408-22.

[26] Roger M, Caliot C, Crouseilles N, Coelho PJ. A hybrid transport-diffusion model for radiative transfer in absorbing and scattering media. J Comput Phys 2014;275:346-62.

[27] Hunter B, Guo Z. Phase-function normalization in the 3-D discrete-ordinates solution of radiative transfer - Part I: Conservation of scattered energy and asymmetry factor. Numerical Heat Transfer, Part B 2012;62:203-22. 


\section{FIGURE CAPTIONS}

Figure 1. Influence of the grid size (a) and quadrature (b) on the incident heat flux, normalized by the emissive power of the hot boundary, on the central line of the top surface.

Figure 2. Incident heat flux, normalized by the emissive power of the hot boundary, along a central line on the top surface of the enclosure.

Figure 3. Influence of the spatial and angular discretizations on the net heat flux on the hot boundary, normalized by its emissive power.

Figure 4. Net heat flux on the hot boundary, normalized by its emissive power, along the axial direction.

Figure 5. Influence of the spatial and angular discretizations on the net heat flux on the hot boundary, normalized by its emissive power.

Figure 6. Net heat flux on the hot boundary, normalized by its emissive power, along the axial direction.

Figure 7. Time history of the dimensionless transmittance and reflectance for test case 3.

Figure 8. Influence of approxiamted scattering phase functions.

(a) Incident heat flux for test case 1.

(b) Net heat flux for test case 2 .

(c) Transmittance for test case 3 .

(d) Reflectance for test case 3 . 

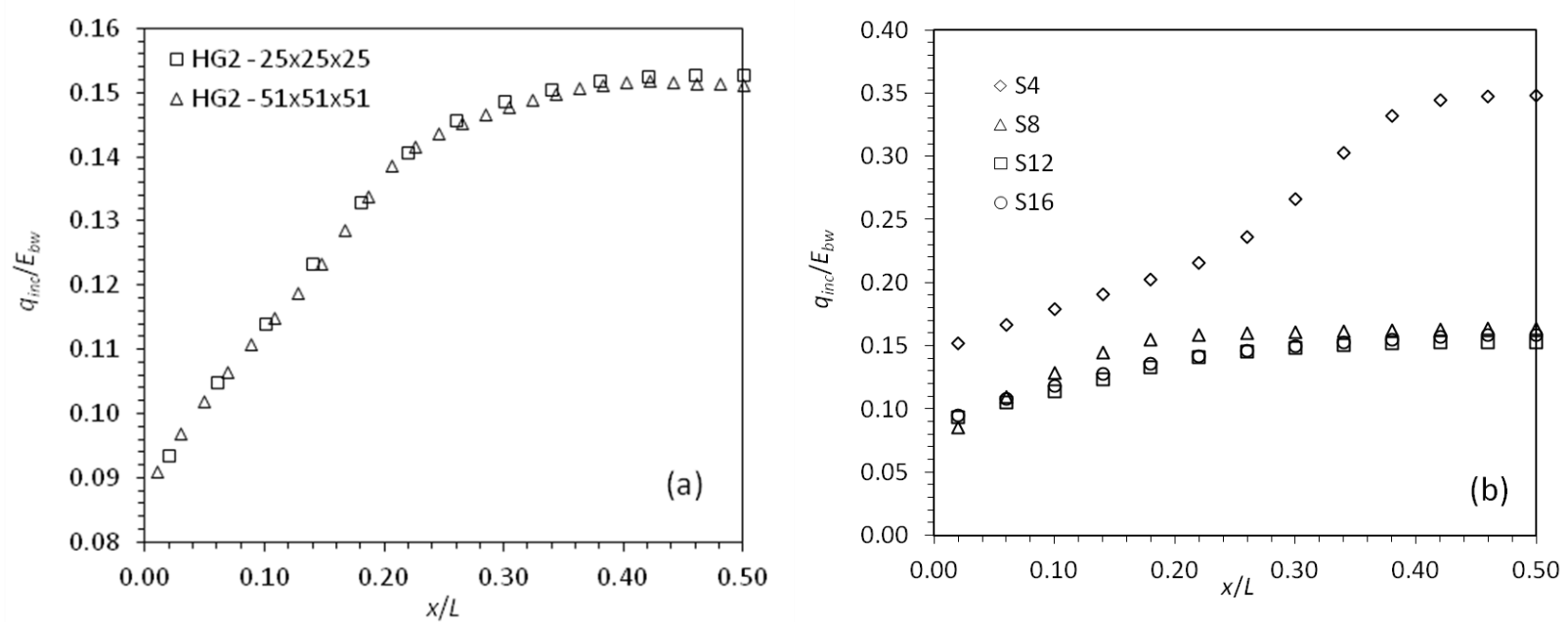

Figure 1. Influence of the grid size (a) and quadrature (b) on the incident heat flux, normalized by the emissive power of the hot boundary, on the central line of the top surface. 


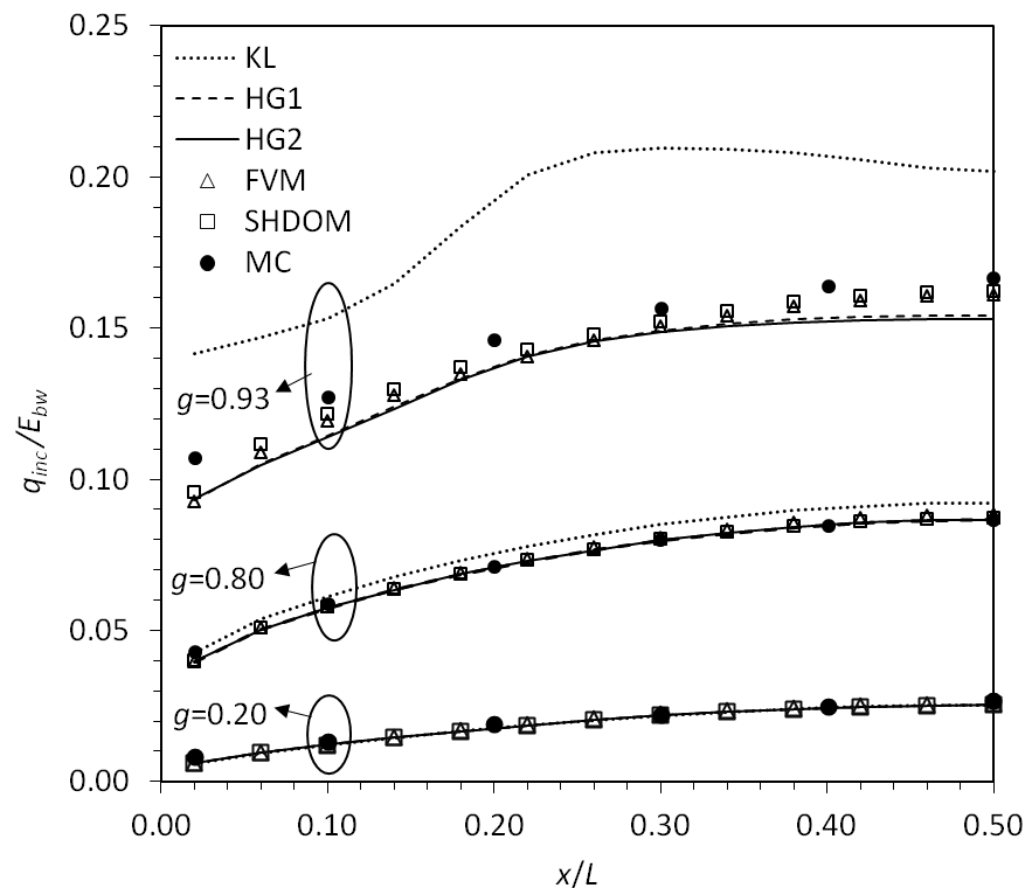

Figure 2. Incident heat flux, normalized by the emissive power of the hot boundary, along a central line on the top surface of the enclosure. 


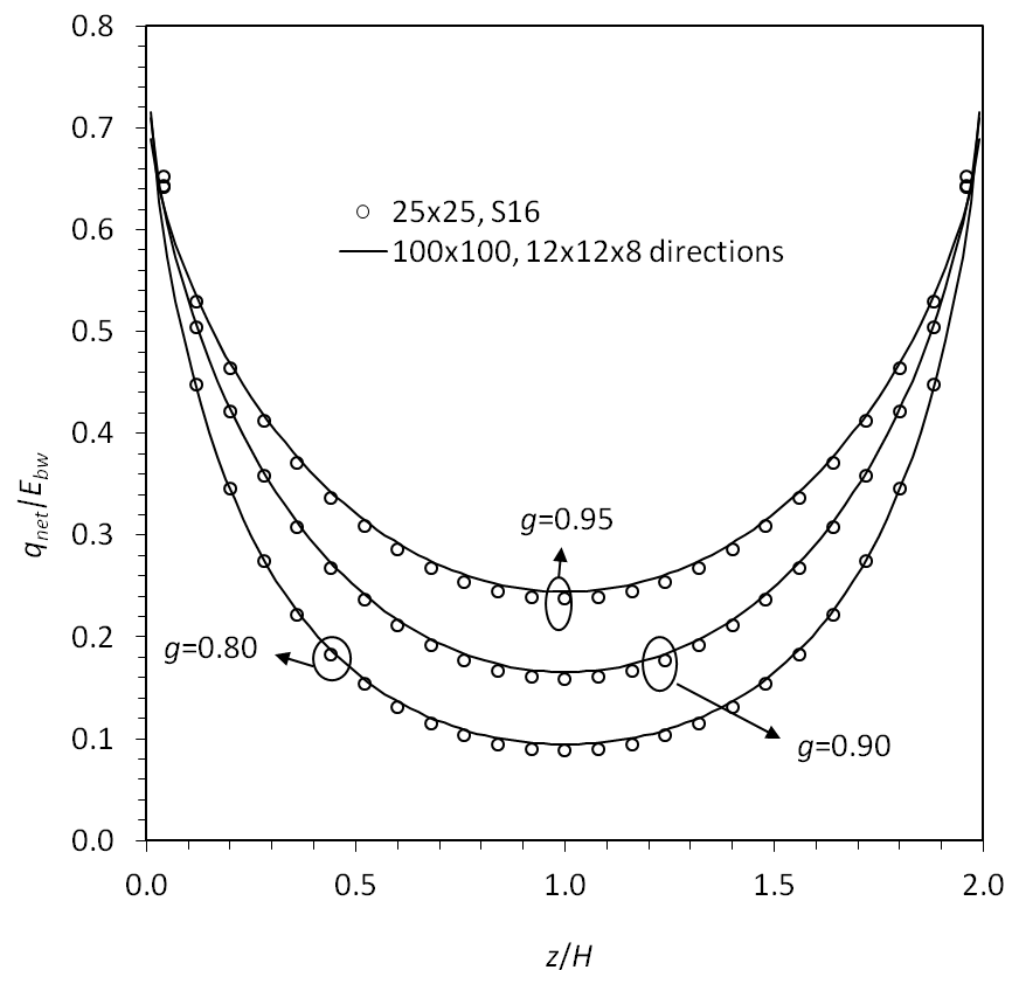

Figure 3. Influence of the spatial and angular discretizations on the net heat flux on the hot boundary, normalized by its emissive power. 


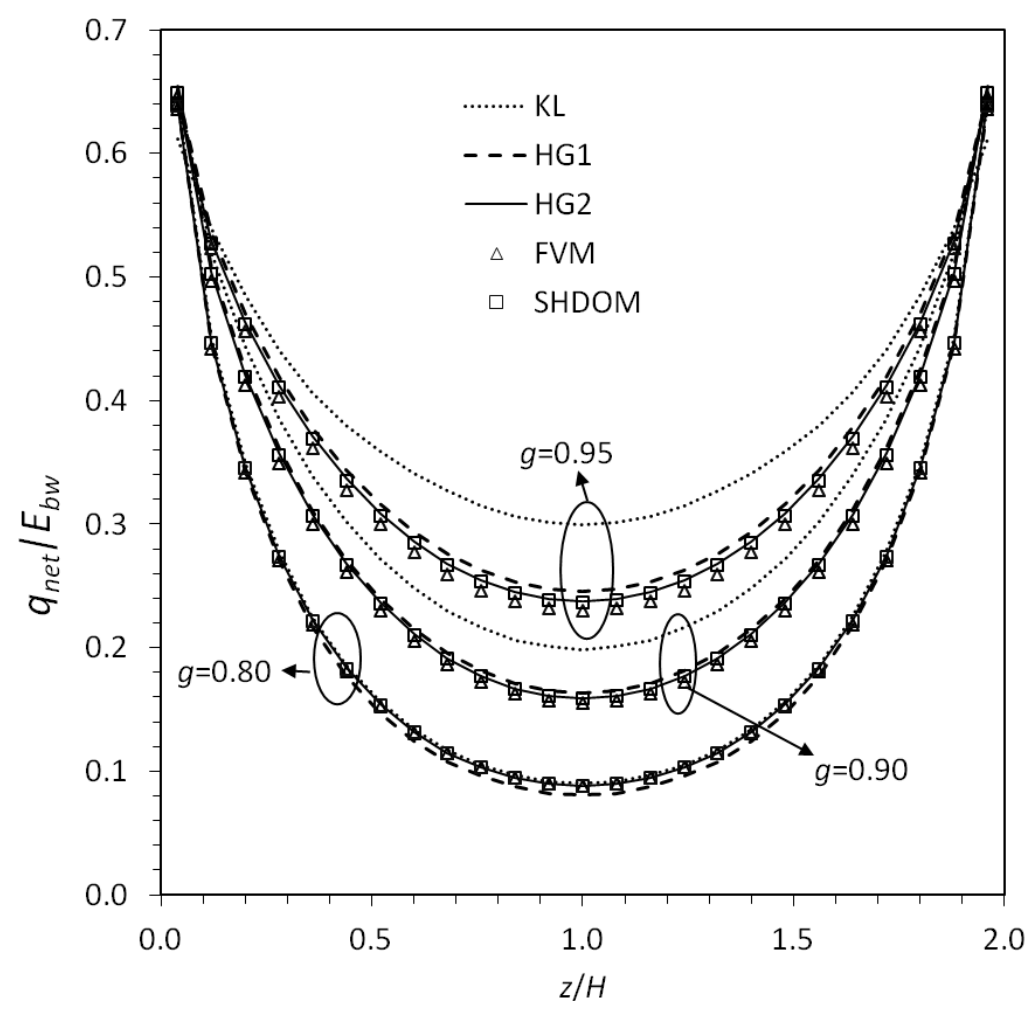

Figure 4. Net heat flux on the hot boundary, normalized by its emissive power, along the axial direction. 


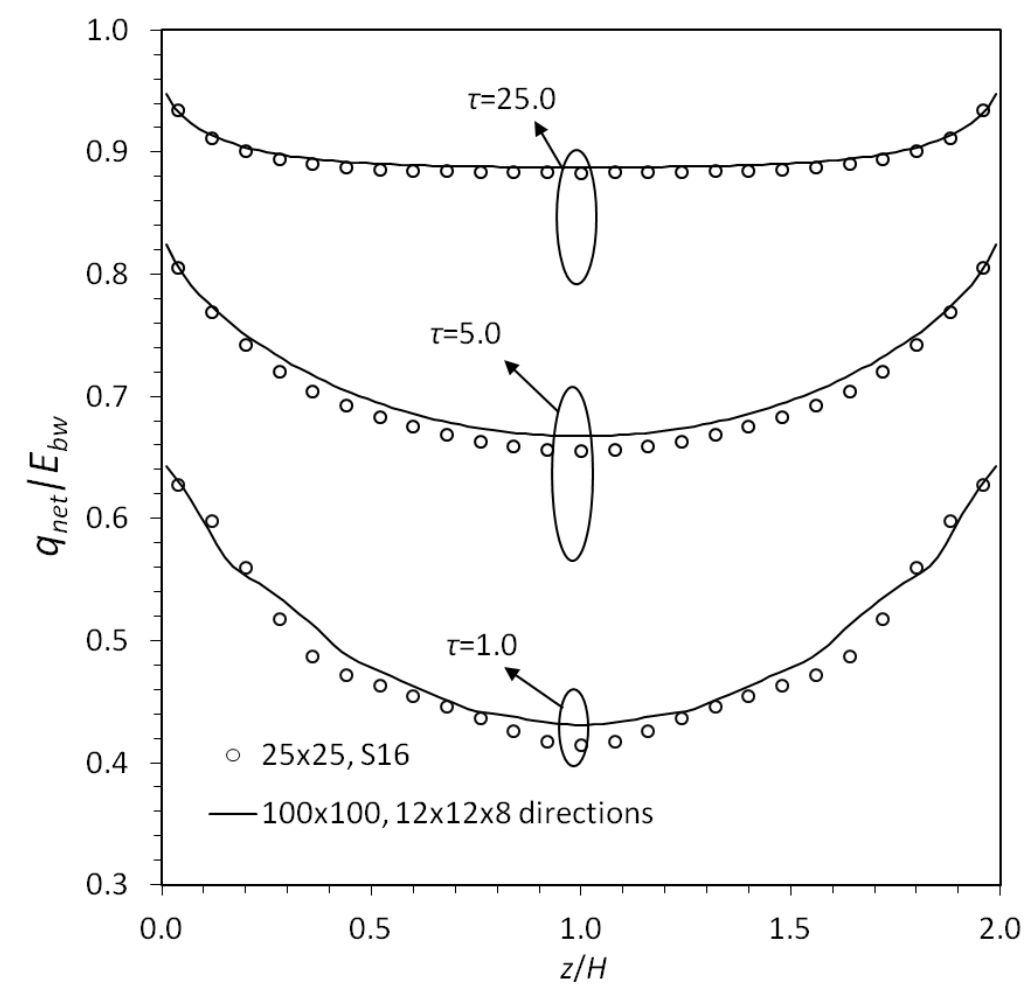

Figure 5. Influence of the spatial and angular discretizations on the net heat flux on the hot boundary, normalized by its emissive power. 


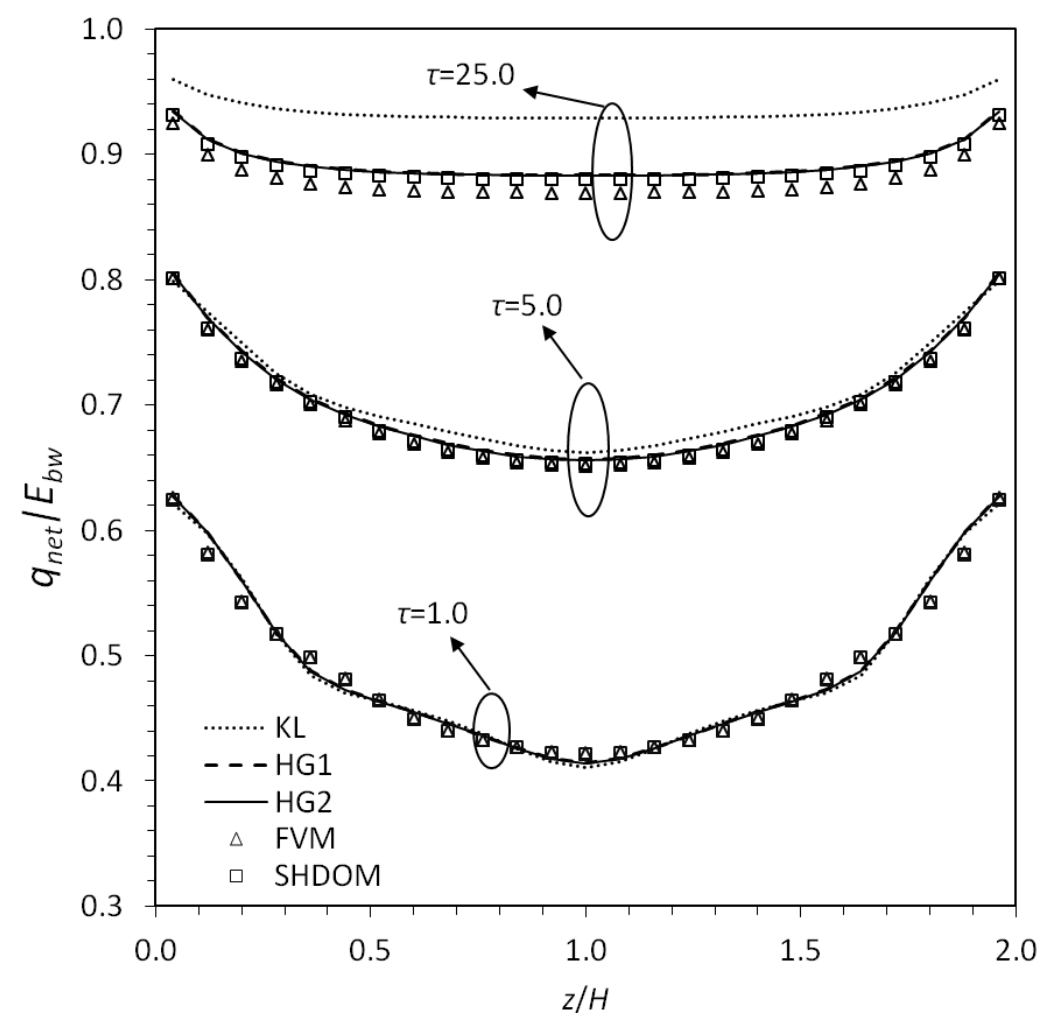

Figure 6. Net heat flux on the hot boundary, normalized by its emissive power, along the axial direction. 

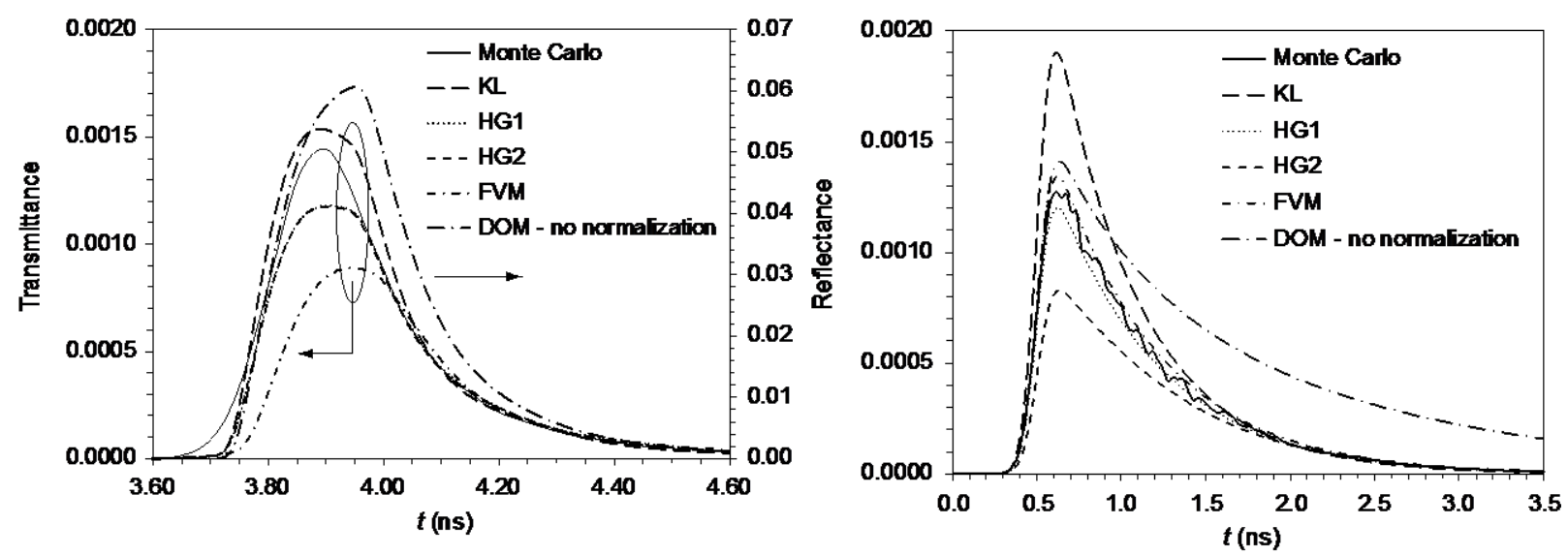

Figure 7. Time history of the dimensionless transmittance and reflectance for test case 3. 

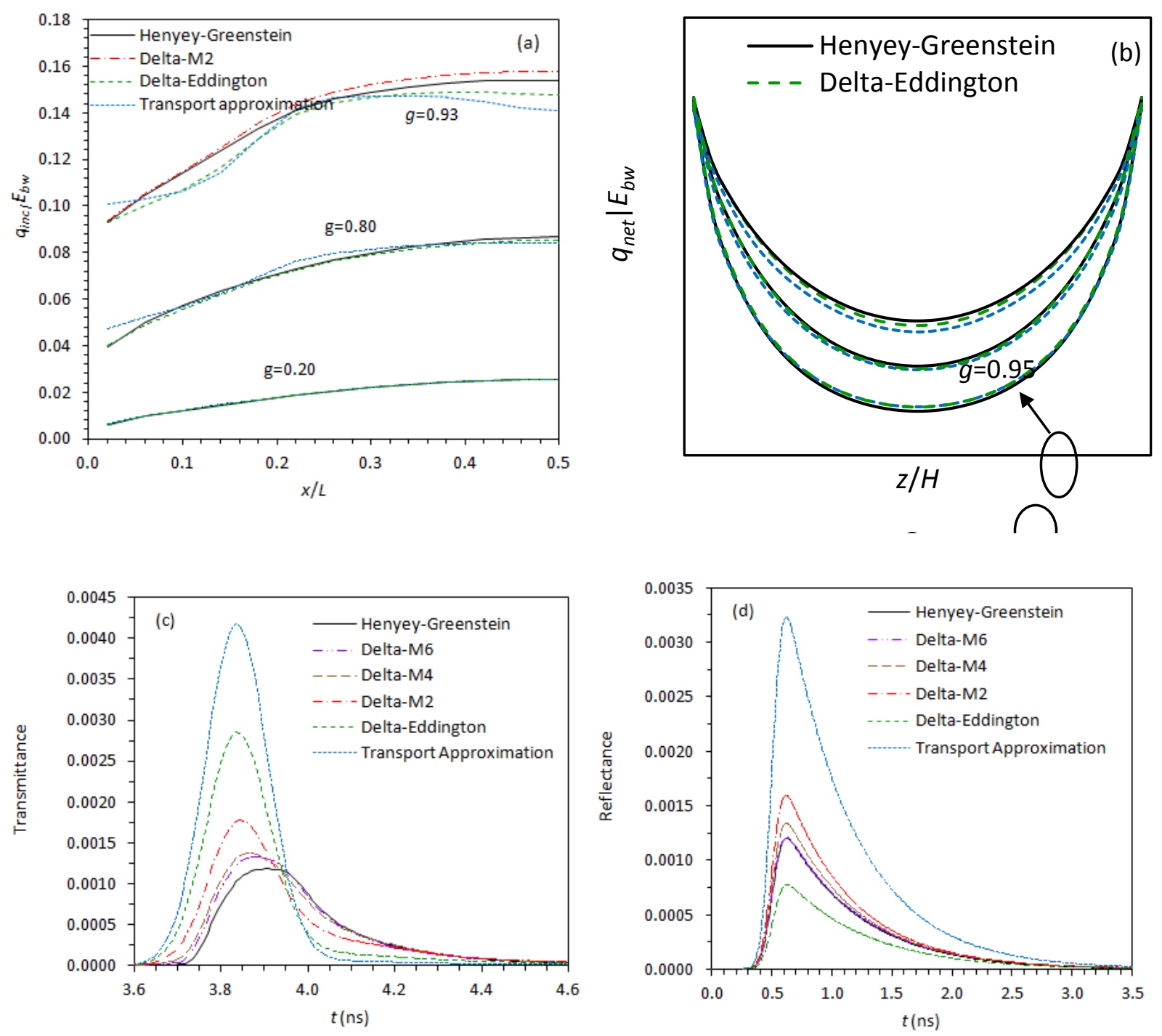

Figure 8. Influence of approxiamted scattering phase functions.

(a) Incident heat flux for test case 1 .

(b) Net heat flux for test case 2 .

(c) Transmittance for test case 3 .

(d) Reflectance for test case 3 . 